\title{
Intravenous administration of Gd-DTPA prior to DWI does not affect the apparent diffusion constant $t^{\text {th }}$
}

\author{
Gang Chen ${ }^{\mathrm{a}, *}$, Sune Nørhøj Jespersen ${ }^{\mathrm{a}}$, Michael Pedersen ${ }^{\mathrm{a}}$, Qi Pang ${ }^{\mathrm{c}}$, \\ Michael R. Horsman ${ }^{\mathrm{b}}$, Hans Stødkilde-Jørgensen ${ }^{\mathrm{a}}$ \\ ${ }^{a}$ MR Research Center, Aarhus University Hospital, Aarhus, DK8200 Aarhus N, Denmark \\ ${ }^{\mathrm{b}}$ Department of Experimental Oncology, Aarhus University Hospital, Aarhus, DK8000 Aarhus C, Denmark \\ ${ }^{\mathrm{c}}$ Department of Neurosurgery, Shandong Provincial Hospital, Shandong University, Jinan, 250021, China \\ Received 9 September 2004; accepted 21 February 2005
}

\begin{abstract}
MRI measurements of water diffusion and blood perfusion are increasingly used for the evaluation of organ functionality and tissue viability (e.g., in tumors). While diffusion-weighted imaging is performed without contrast agents, measurement of blood perfusion is normally performed based on the administration of paramagnetic substances such as gadolinium diethylenetriamine pentaacetic acid (GdDTPA). Simultaneous measurements of these two parameters are often preferred. However, it may be argued that Gd-DTPA causes constriction of small blood vessels or alters hemodynamic parameters such as blood viscosity, thereby corrupting subsequent measurements of the apparent diffusion constant (ADC). The objective of the current study was to investigate the possible changes in the ADC in tumors following intravenous administration of 0.2 and $0.4 \mathrm{mmol} / \mathrm{kg}$ of Gd-DTPA in mice. The study was conducted with C3H mouse mammary carcinomas inoculated in the right foot of the animal subjects. The results were compared with findings in a sham group, demonstrating that Gd-DTPA had no significant impact on the ADC as measured in a 7-T animal system.

(C) 2005 Elsevier Inc. All rights reserved.
\end{abstract}

Keywords: MRI; Diffusion-weighted imaging; Gadolinium-DTPA; C3H mouse mammary carcinoma

\section{Introduction}

Diffusion-weighted imaging (DWI) and perfusionweighted imaging (PWI) are important methods for the evaluation of functionality and viability in various organs. Furthermore, DWI and PWI are routinely used for the assessment of tissue response to therapy.

PWI relies on the hemodynamic dispersion of a paramagnetic contrast agent, such as gadolinium diethylenetriamine pentaacetic acid (Gd-DTPA), rapidly introduced into the blood. As a result of its influence on the local magnetic susceptibility and the relaxation rates of water, a transient change in the MR signal can be observed during bolus passage, thereby facilitating estimation of regional blood volume, regional blood flow and mean transit time.

\footnotetext{
This study was funded in part by the Danish Medical Research Council (Grant No. 22-05-0519 BMP-MP) and the Danish Cancer Society.

* Corresponding author. Tel.: +45 89495264; fax: +45 89496004.

E-mail address: gangchen@mr.au.dk (G. Chen).
}

Thus, PWI has become an important method for functional evaluation of organs and is increasingly used as an indicator for pathological conditions (e.g., stroke in the brain). However, since Gd-DTPA is excreted exclusively through glomerular filtration with a mean half-life of approximately $70 \mathrm{~min}$ in the serum [1], subsequent imaging procedures may occasionally become affected by the systemic presence of such a contrast agent.

DWI is known to reflect the inherent molecular tumbling of water in tissues and does not require administration of a contrast agent. Brownian motion in the presence of magnetic field gradients leads to a diffusion-dependent dephasing of individual proton magnetic moments, which reduces the signal intensity in diffusion-weighted images. The apparent diffusion constant (ADC) calculated from two or more diffusion-weighted images is sensitive to intracellular and extracellular volume fractions, cell sizes and membrane permeability. Consequently, changes in the ADC may indicate a shift in the distribution of water between the two compartments as 
observed in tissues with cellular swelling. In clinics, DWI plays an important role in the assessment of a wide variety of diseases such as brain neoplasms, intracranial infections, brain injury, acute ischemic stroke and differentiation of cytotoxic and extracellular edemas. In addition, it has become useful for identifying tumor grade and regions of hyperactive cells [2].

DWI and PWI are usually used separately, although data provided by each of them are to some extent complementary. However, significant interest has recently appeared for conducting DWI and PWI consecutively especially in the evaluation of stroke [3]. In situations where the MRI protocol dictates DWI to be performed before PWI, there is no risk of interference between the two measurements. However, if measurements are repeated within approximately $1 \mathrm{~h}$, remaining Gd-DTPA in the blood may interfere with subsequent measurements of the ADC. The present study aimed to evaluate the possible effects of Gd-DTPA administration using doses relevant for PWI on ADC values as measured in a high-field scanner in animal tumors.

\section{Materials and methods}

\subsection{Animal model}

$\mathrm{C} 3 \mathrm{H}$ mouse mammary carcinomas were transplanted on the right rear foot of 10-week to14-week-old female CDF1 mice as previously described in detail [4]. Experiments were carried out when the tumors reached a volume of $200 \mathrm{~mm}^{3}$ approximately 3 weeks after implantation. The tumor volume was determined by the following formula: $\mathrm{D} 1 \times \mathrm{D} 2 \times \mathrm{D} 3 \times \pi / 6$, where $\mathrm{D} 1, \mathrm{D} 2$ and D3 represent three orthogonal diameters. The animals were awake during the entire procedure. A 25-G venflon connected with a polythene tube $(\varnothing, 0.38 \mathrm{~mm})$ was inserted into a tail vein. The animals were restrained in a plastic jig (Fig. 1). Their right foot was exposed and secured with tape, and their tail was put through a hole in the rear of the jig,

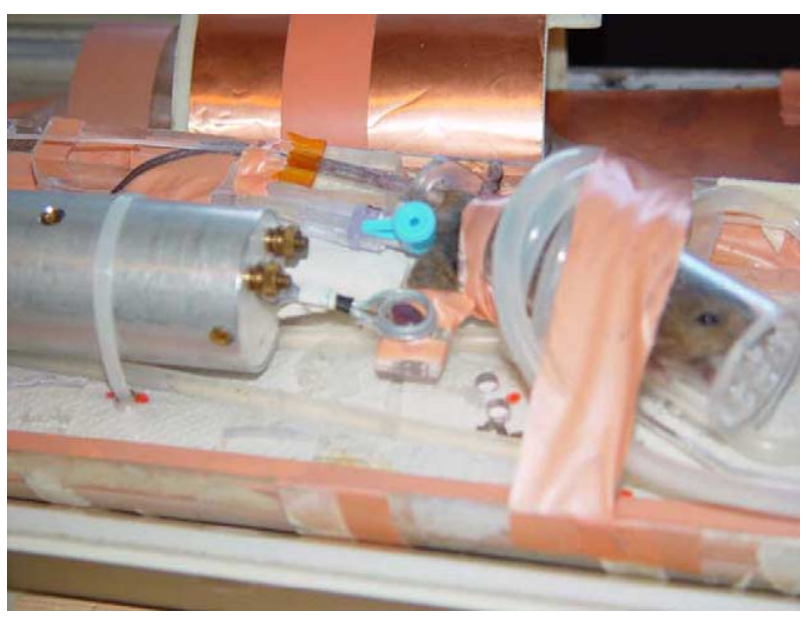

Fig. 1. The experimental setup with a mouse in a jig. helping secure each mouse in a fixed position. The tumor was positioned adjacent to a radio frequency surface coil. To ensure that the mice maintained a body temperature of $37 \pm 0.5^{\circ} \mathrm{C}$ (rectal temperature was monitored) throughout the experiment, a plastic tube with circulating warm water was positioned around their bodies. MRI was initiated 30 min after each mouse had been inserted into the magnet.

Twenty-four animals were divided into four groups. Group $1(n=3)$ consisted of mice used to test that an injection of $0.2 \mathrm{mmol} / \mathrm{kg}$ of Gd-DTPA entered the intravascular system. Dynamic contrast-enhanced MRI was performed to confirm the presence of Gd-DTPA in the blood. Group $2(n=7)$ served as a control group - a catheter was inserted but no contrast agent was administered in the animals. Group $3(n=8)$ received a dose of $0.2 \mathrm{mmol} / \mathrm{kg}$ of Gd-DTPA and, finally, Group $4(n=6)$ received a dose of $0.4 \mathrm{mmol} / \mathrm{kg}$ of Gd-DTPA.

DWI and T2 measurements were performed at $-30,0$, 60 and 120 min following administration of Gd-DTPA. The experimental protocol was approved by the local committee for animal welfare and the experiments conformed to Danish- and European Union-approved guidelines for animal welfare.

\section{2. $M R I$}

A 7-T horizontal bore magnet (Oxford Instruments, Oxford, UK) interfaced to a Unity Inova console (Varian, Palo Alto, CA, USA) and equipped with a $12.5-\mathrm{G} / \mathrm{cm}$ gradient system (Tesla Engineering, West Sussex, UK) was used. A home-built 9-mm-diameter double-turned surface coil served as both transmitter and receiver.

DWI was performed with a Stejskal-Tanner spin-echo imaging sequence [5] using the following parameters: TR of $2.3 \mathrm{~s}$, TE of $0.05 \mathrm{~s}$, a slice thickness of $2 \mathrm{~mm}, 128 \times 128$ data matrix, coronal orientation and a field of view of $3 \times 3 \mathrm{~cm}^{2}$; one slice was acquired. The ADC was calculated pixelwise as $\mathrm{ADC}=\ln [S(0) / S(b)] / b$, where $S(b)$ is the signal intensity at diffusion-weighting factor $b$ and $S(0)$ is the signal intensity at $b=0$. The factor $b$ was given by $b=\gamma^{2} \delta^{2}(\Delta-\delta / 3) G^{2}$, where $\gamma$ is the gyromagnetic constant; $\delta$, the duration of the individual gradient pulses; $\Delta$, the time separation between the leading edges of the two gradient pulses; and $G$, the strength of the diffusion gradient, which was applied in the readout direction. Diffusion gradients equivalent to $b$ values of 0 and $1370 \times 10^{-3} \mathrm{~s} / \mathrm{mm}^{2}$ were used using gradient pulse widths of $\delta=15 \mathrm{~ms}$ and $\Delta=30 \mathrm{~ms}$.

The T2-weighted images were acquired using a spinecho sequence with the following parameters: TR of $2.3 \mathrm{~s}$, TE of $0.05 \mathrm{~s}$, a slice thickness of $2 \mathrm{~mm}, 128 \times 128$ data matrix, coronal orientation and field of view $3 \times 3 \mathrm{~cm}^{2}$; two images were averaged.

The dynamic contrast-enhanced MRI used on Group 1 was performed with a T1-weighted gradient-spoiled gradient-echo sequence with the following parameters: TR of $0.03 \mathrm{~s}$, TE of $0.003 \mathrm{~s}$, a flip angle of $40^{\circ}$, a slice thickness of 
$2 \mathrm{~mm}, 64 \times 64$ data matrix, coronal orientation and a field of view of $3 \times 3 \mathrm{~cm}^{2}$; one slice was acquired.

\subsection{Image analysis}

Image postprocessing was performed with ImageJ, a freely available program developed by Wayne Rasband of the National Institute of Health (http://rsb.info.nih.gov/ij). Regions of interest were manually drawn on the T2-weighted images encompassing the entire tumor and duplicated on the calculated ADC maps.

The contrast-to-noise ratio (CNR) was calculated on the T2-weighted images using the following equation: $\mathrm{CNR}=($ intensity (tumor) - intensity(tissue))/intensity(noise).

Statistical analysis was performed with Matlab (The MathWorks, Natick, MA, USA) using one-way analysis of variance (ANOVA). The Lilliefors-modified KolmogorovSmirnov test was applied to evaluate data distribution. A few of the samples were marginally nonnormal, for those we used the nonparametric Kruskal-Wallis test; for all the others, we used ANOVA. We used a statistical significance level of .05 .

Image postprocessing of dynamic contrast-enhanced MRI was performed using software written in IDL (Research Systems, Boulder, CO, USA).

\section{Results}

The measurements of Group 1 served to verify the presence of Gd-DTPA in the blood after intravenous administration of $0.2 \mathrm{mg} / \mathrm{kg}$ of Gd-DTPA. Fig. 2 demonstrates changes in signal intensity, indicating that the contrast agent was successfully injected into the blood.

Fig. 3A shows the results for the control group plotted as means with S.D.s, demonstrating that ADC values

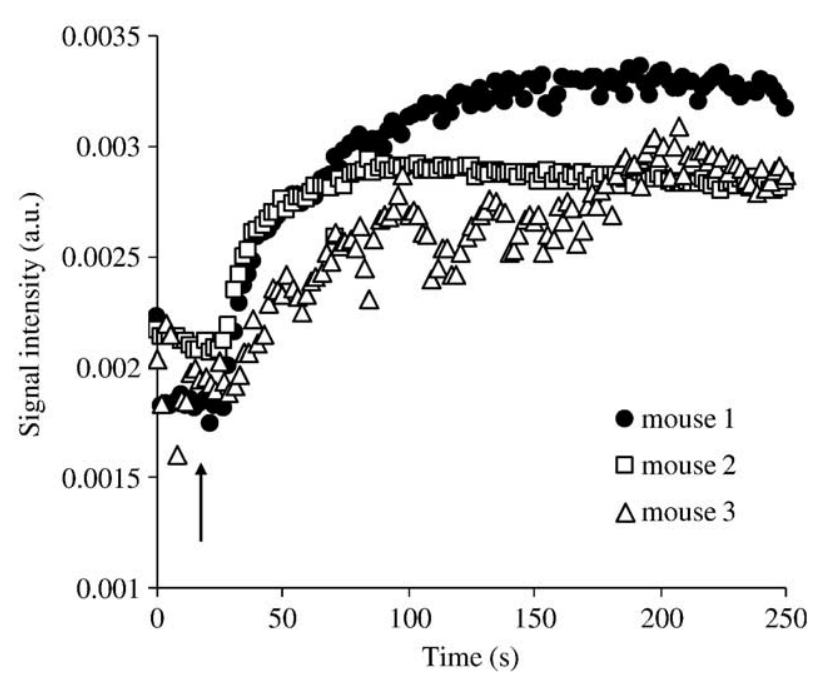

Fig. 2. Signal change in T1-weighted images of tumor tissue from three mice after administration of $0.2 \mathrm{mmol} / \mathrm{kg}$ of Gd-DTPA (arrow). The immediate increase in the signal indicates that Gd-DTPA was successfully given to the blood.
A

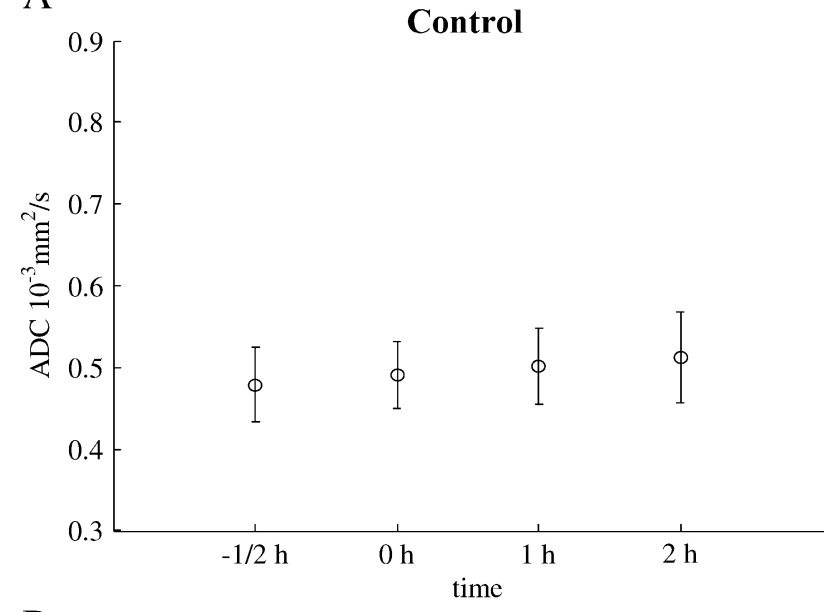

B

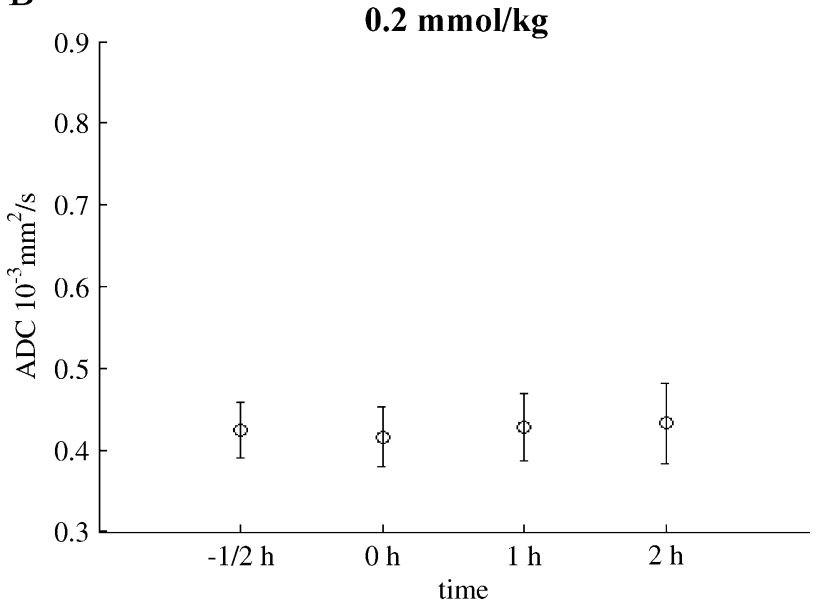

C

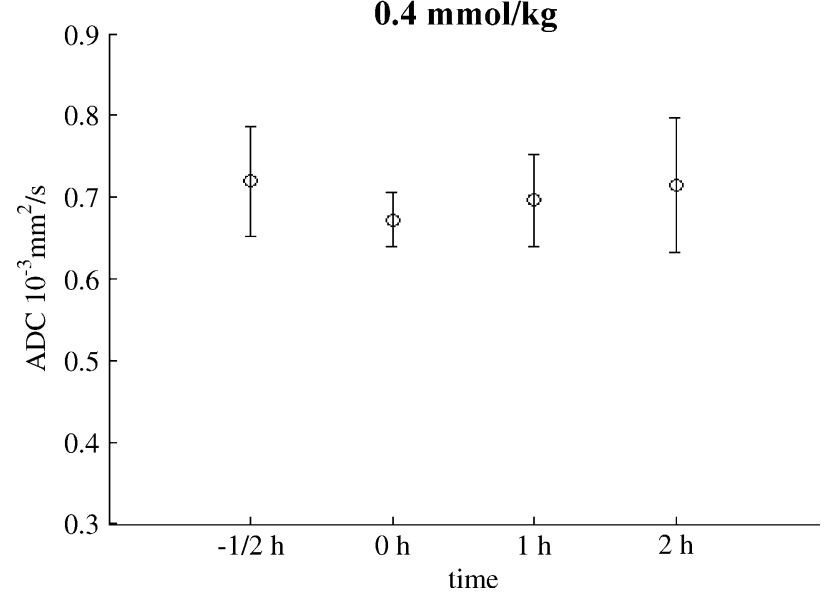

Fig. 3. (A) ADC of the tumor in the control group (Group 2). (B) ADC of the tumor in mice receiving $0.2 \mathrm{mmol} / \mathrm{kg}$ of Gd-DTPA (Group 3). (C) ADC of the tumor in mice receiving $0.4 \mathrm{mmol} / \mathrm{kg}$ of Gd-DTPA (Group 4). Gd-DTPA is injected immediately before $0 \mathrm{~h}$.

at $-30,0,60$ and $120 \mathrm{~min}$ are comparable. This was confirmed by one-way ANOVA $(P=.57)$.

Likewise, in animals receiving $0.2 \mathrm{mmol} / \mathrm{kg}$ of Gd-DTPA (Group 3), one-way ANOVA did not reveal significant differences between ADC measurements performed before 
and after Gd-DTPA injection ( $P=.85$ Kruskal-Wallis test) (Fig. 3B). Similarly, in animals receiving $0.4 \mathrm{mmol} / \mathrm{kg}$ of Gd-DTPA (Group 4), one-way ANOVA did not reveal significant differences between ADC measurements $(P=.77$ ANOVA) (Fig. 3C).

Another issue of diagnostic relevance concerns the influence of Gd-DTPA on the CNR of tumors. CNR was measured on T2-weighted images. Again, the result of the

A

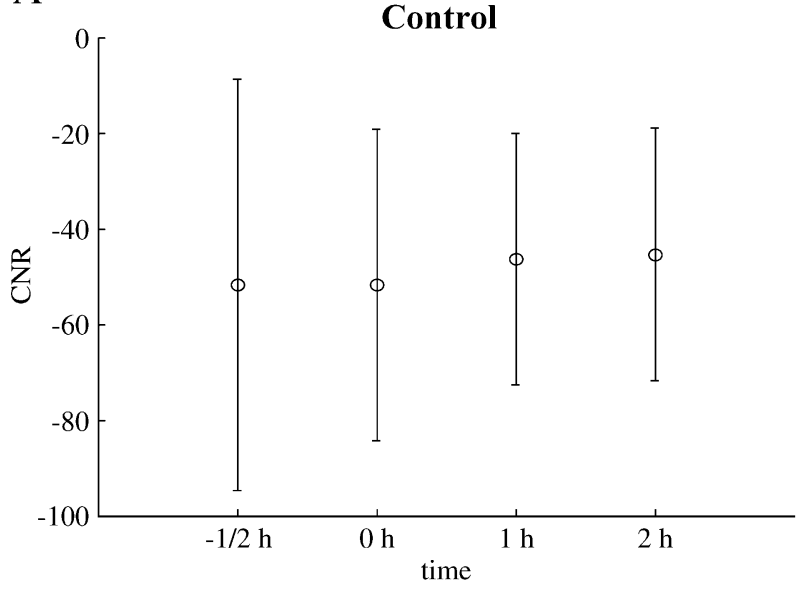

B

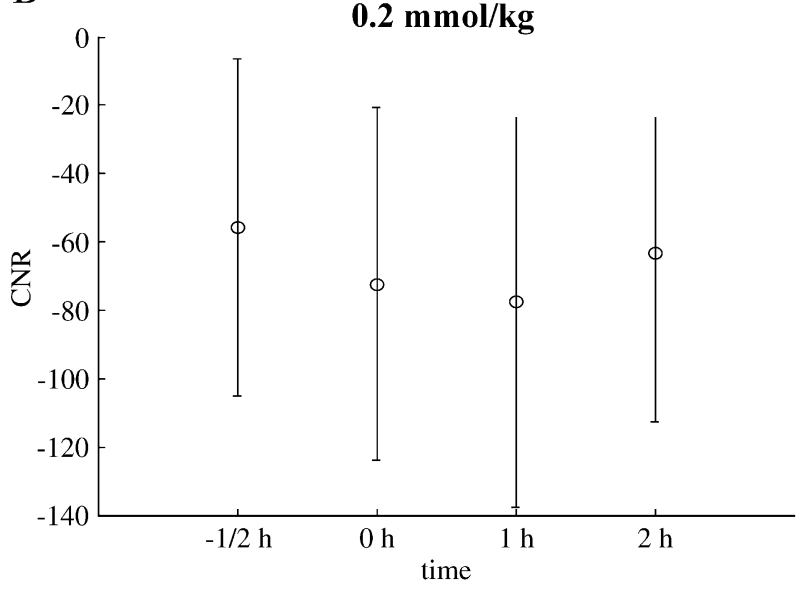

$\mathrm{C}$

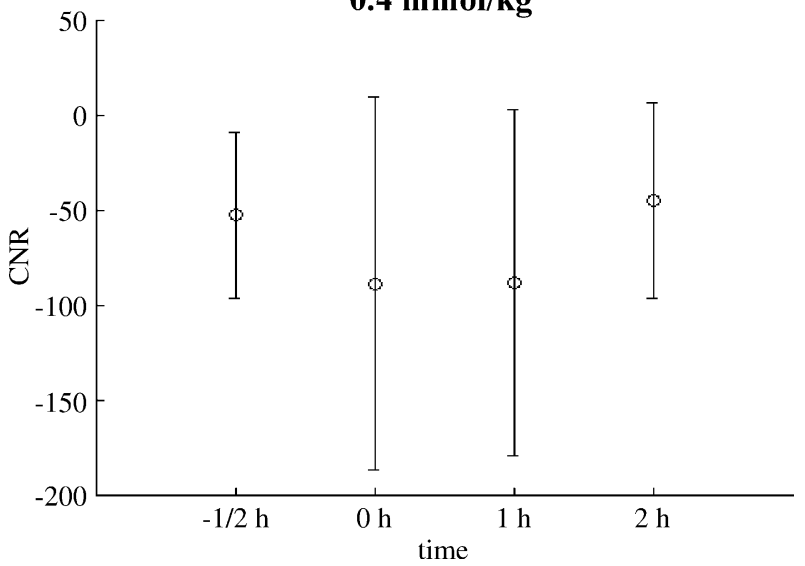

Fig. 4. The CNRs of tumors in the control group (A), $0.2 \mathrm{mmol} / \mathrm{kg}$ group (B) and $0.4 \mathrm{mmol} / \mathrm{kg}$ group (C). Gd-DTPA is injected immediately before $0 \mathrm{~h}$.
Lilliefors-modified Kolmogorov-Smirnov test was consistent with the assumption that the T2 values follow a gaussian distribution. The mean CNR with S.D. is shown in Fig. 4, demonstrating that injection of Gd-DTPA had no significant effect on CNR (control group, $P=.98$ KruskalWallis test; Group 3, $P=.85$ Kruskal-Wallis test; Group 4, $P=.64$ ANOVA).

\section{Discussion}

The aim of this study was to evaluate the effect of intravenous injection of Gd-DTPA on the ADC. The main results demonstrated that although a relatively high dose of Gd-DTPA was given, the ADC did not change, in agreement with the results obtained by Morriss and Haselgrove [6] and Fitzek et al. [7].

The results from Group 1 indicated a successful injection of Gd-DTPA by an observed increase in the T1-weighted signal intensity in the tumor. The results from Group 2, the control group, served as a reassurance that tumor ADC and CNR did not change as a result of conditions besides the Gd-DTPA bolus injection.

In contrast to the results presented here, some authors [8-10] found that administration of Gd-DTPA $(0.2 \mathrm{mmol} / \mathrm{kg})$ in humans reduced the ADC by $2.4 \%$. Likewise, Yamada et al. [11] found a $1.3 \%$ reduction of the ADC after injection of $0.1 \mathrm{mmol} / \mathrm{kg}$ of Gd-DTPA. The work of both research groups were performed on 1.5-T clinical scanners. Yamada et al. [11] suggested that the observed decrease in the ADC could be explained by a decrease in the intravascular compartment's contribution to the ADC value. This explanation is implicitly based on the assumption that the ADC can be interpreted as arising from two components corresponding to the intracellular and extracellular spaces.

The discrepancy between the present and previously published results may likely be explained by the following differences. First, our experiments were performed on a 7-T MRI system whereas previous studies used clinical 1.5-T magnets. The relaxation time $\left(T_{i}\right)$ is reciprocally related to the concentration of Gd-DTPA by the following equation: [Gd-DTPA] $=\left(1 / T_{i}-1 / T_{i(0)}\right) / R_{i}$, where $R_{i}$ is the specific relaxivity of Gd-DTPA, valid for both longitudinal $(i=1)$ and transverse $(i=2)$ relaxation. The constant $T_{i(0)}$ is the $\mathrm{T} 1$ or T2 value in the absence of Gd-DTPA. It is well known that the relaxation rates of liquids are different at low and high magnetic fields. This may be explained from the theoretical arguments described by Caravan et al. [12], implying that $R_{i}$ can be dramatically changed at higher field strengths with the result that the Gd-DTPA-induced change in T2 (and T1) is relatively lower at a high magnetic field strength. Taking into account the inherent T2 dependence of the Stejskal-Tanner diffusion-weighted sequence, it may be argued that the relatively larger Gd-DTPA-induced reduction of extracellular T2 at low magnetic field strengths may affect the ADC more than would that at high magnetic field strengths. The observed influence of the ADC after injection 
of Gd-DTPA as reported by previous authors may consequently be interpreted as a result of a T2 contamination and not as a result of a physical change in the diffusion characteristics of the tissue. Second, differences in the experimental setup, such as pulse sequence and $b$ values, may also account for the disagreement as well as the fact that the biologic tissues being investigated here are mouse carcinomas whereas the previously mentioned studies focused on human tissues.

In conclusion, we found that the injection of above-normal doses of Gd-DTPA did not affect the ADC value of mouse mammary carcinomas. In addition, we also found that GdDTPA had no effect on the CNR of tumors in T2-weighted images. The consequence of these results is the possibility of performing PWI and DWI consecutively and in any order, bearing in mind that our studies were performed at a relatively high magnetic field strength and that similar conclusions may not necessarily be drawn on clinical magnets. Likewise, we stress that higher concentrations of Gd-DTPA may ultimately interfere with the molecular diffusion of water, leading to a measurable effect on the ADC.

\section{References}

[1] Van Wagoner M, Worah D. Gadodiamide injection. First human experience with the nonionic magnetic resonance imaging enhancement agent. Invest Radiol 1993;28(Suppl 1):S44-8.

[2] den Boer JA, Folkers PJM. MR perfusion and diffusion imaging in ischaemic brain disease. Medicamundi 1997;41:20-35.
[3] Ostergaard L, Sorensen AG, Chesler DA, Weisskoff RM, Koroshetz $\mathrm{WJ}$, $\mathrm{Wu} \mathrm{O}$, et al. Combined diffusion-weighted and perfusionweighted flow heterogeneity magnetic resonance imaging in acute stroke. Stroke 2000;31(5):1097-103.

[4] Overgaard J. Simultaneous and sequential hyperthermia and radiation treatment of an experimental tumor and its surrounding normal tissue in vivo. Int J Radiat Oncol Biol Phys 1980;6:1507-17.

[5] Stejskal EO, Tanner JE. Spin diffusion measurements: spin echoes in the presence of a time dependent field gradient. J Chem Phys 1965; 42:288-92.

[6] Morriss C, Haselgrove J. Intravenous gadolinium does not alter the apparent diffusion coefficient: a study in enhancing brain tumors. In: Book of abstracts: Eighth Annual Meeting of the International Society of Magnetic Resonance in Medicine, Denver: ISMRM; 2000. p. 1103.

[7] Fitzek C, Mentzel HJ, Fitzek S, Sauner D, Kaiser WA, Reichenbach JR. Echoplanar diffusion-weighted MRI with intravenous gadoliniumDTPA. Neuroradiology 2003;45:592-7.

[8] Does MD, Zhong J, Gore JC. In vivo measurement of ADC change due to intravascular susceptibility variation. Magn Reson Med 1999;41: $236-40$.

[9] Zhong J, Kennan RP, Gore JC. Effect of susceptibility variations on NMR measurements of diffusion. J Magn Reson 1991;95:267-80.

[10] Zhong J, Kennan RP, Fulbright RK, Gore JC. Quantification of intraand extravascular contributions to BOLD effects induced by alteration of oxygenation or intravascular contrast agent. Magn Reson Med 1998;40:526-36.

[11] Yamada K, Kubota H, Kizu O, Nakamura H, Ito H, Yuen S, et al. Effect of intravenous gadolinium-DTPA on diffusion-weighted images: evaluation of normal brain and infarcts. Stroke 2002;33: 1799-802.

[12] Caravan P, Ellison JJ, McMurry TJ, Lauffer RB. Gadolinium(III) chelates as MRI contrast agents: structure, dynamics, and applications. Chem Rev 1999;99:2293-352. 